\title{
Anti-aging effects of high molecular weight proteoglycan from salmon nasal cartilage in hairless mice
}

\author{
MASASHI GOTO ${ }^{1,4}$, SHOTA YAMAZAKI $^{2}$, YOJI KATO $^{3}$, KAZUSHI YAMAMOTO ${ }^{4}$ and YOHTARO KATAGATA $^{1,2}$ \\ ${ }^{1}$ Science of Bioresources, The United Graduate School of Agricultural Sciences, Iwate University, Morioka; \\ ${ }^{2}$ Department of Biochemistry and Biotechnology, Faculty of Agriculture and Life Science; ${ }^{3}$ Laboratory of Food \\ Science, Faculty of Education, Hirosaki University, Hirosaki; ${ }^{4}$ R\&D Department, Sunstar Inc., Takatsuki, Japan
}

Received December 21, 2011; Accepted February 2, 2012

DOI: $10.3892 /$ ijmm.2012.918

\begin{abstract}
Proteoglycans comprise a family of complex macromolecules consisting of a core protein with covalently attached glycosaminoglycan (GAG) chains. The skin antiaging effects of oral administration of proteoglycan fractions with different molecular weights from salmon nasal cartilage were investigated in a hairless mouse model of skin aging; aging was caused by repeated ultraviolet B (UVB) irradiation. Three proteoglycan fractions of different molecular weights were prepared from salmon nasal cartilage water extract by ion-exchange column chromatography and gel filtration column chromatography. Physiological and histological analysis of the skin indicated that oral administration of high molecular weight proteoglycan inhibited UVB-induced skin aging, defined as increased erythema, increased transepidermal water loss (TEWL), decreased hydration, and epidermal and dermal hypertrophies. The serum and dorsal skin inflammatory cytokine levels indicated that high molecular weight proteoglycan acts on gut immunity and improves skin by inhibiting surplus inflammatory cytokines produced by UVB irradiation. These results suggest that high molecular weight proteoglycan from salmon nasal cartilage is effective in preventing skin aging.
\end{abstract}

\section{Introduction}

With the aging of the population, skin anti-aging is becoming increasingly important for the maintenance of health and for a high quality of life. Skin aging can be attributed to chronological aging and UV-induced photoaging. In particular, aging of the facial skin is markedly influenced by photoaging. Skin

Correspondence to: Professor Yohtaro Katagata, Department of Biochemistry and Biotechnology, Faculty of Agriculture and Life Science, Hirosaki University, 3 Bunkyo-cho, Hirosaki 036-8561, Japan

E-mail: katagata@cc.hirosaki-u.ac.jp

Key words: proteoglycan, salmon nasal cartilage, skin, anti-aging, hairless mouse reverts to a low level of epidermal barrier function when irradiated by UV, and shows markedly increased transepidermal water loss (TEWL), which eventually results in dry skin $(1,2)$. In addition, UV-induced epidermal and dermal hypertrophies result in the stiffening of the skin and the formation of wrinkles (3). Therefore, the progression of skin aging can be suppressed by preventing UV-induced photoaging.

The effects of topical application of various agents on photoaging have been extensively studied, but there have been only a few studies on the impact of oral treatments on photoaging. For example, the effects of the oral intake of various compounds in defense against skin damage caused by UV have been examined. Oral administration of collagen peptide was reported to induce an increase in fibroblast density and to enhance the formation of dermal collagen fibrils in piglet skin (4), as well as to suppress the UVB-induced decreases in skin hydration, epidermal hypertrophy, and amount of soluble type I collagen in mouse skin (5). Oral intake of soy isoflavones was shown to suppress UVB-induced matrix metalloproteinase expression and the subsequent collagen degradation in mouse skin (6). In addition, oral intake of green tea polyphenols was shown to prevent UVB-induced protein oxidation and matrix metalloproteinase expression in mouse skin (7).

Proteoglycans comprise a family of complex macromolecules consisting of a core protein with covalently attached glycosaminoglycan (GAG) chains (8). The physiological activity of a proteoglycan from salmon nasal cartilage has been investigated, and several studies have shown that oral administration of salmon nasal cartilage proteoglycan suppresses the progression of colitis $(9,10)$. In our previous study $(11)$, we demonstrated that oral intake of salmon nasal cartilage extract (SNCE), including abundant proteoglycan, in an experimental animal model of photoaging has an anti-aging effect on the skin. However, the active ingredient of SNCE could not be identified as a crude extract was used.

In the present study, to confirm whether the proteoglycan of SNCE contributes to the anti-aging effect on skin, we purified fractions of proteoglycan with different molecular weights from salmon nasal cartilage by DEAE-Sephacel ion-exchange and Sepharose CL-2B gel filtration column chromatography. We evaluated the anti-aging effects of oral administration of these proteoglycan fractions in an experimental animal model of photoaging. 


\section{Materials and methods}

Preparation of salmon nasal cartilage extract (SNCE). SNCE was prepared from the nasal cartilage of salmon heads as previously reported (11). That is, nasal cartilage from salmon heads was crushed using a meat chopper, then suspended in 1-2 volumes of water and centrifuged at 8,000 rpm for $30 \mathrm{~min}$ at room temperature. The residues were then collected. This procedure was performed twice. The residues thus obtained were lyophilized and crushed finely using a powdering machine (MKA-2J; Masuko Sangyo Co. Ltd., Saitama, Japan). Powdered residues were suspended in 10 volumes of ethanol and centrifuged at 3,000 rpm for $15 \mathrm{~min}$ at room temperature; precipitations were air-dried to obtain powders. These defatted powders were suspended at a concentration of $2 \%$ in water and centrifuged at $15,000 \mathrm{rpm}$ for $30 \mathrm{~min}$ at $4^{\circ} \mathrm{C}$. After centrifugation, the supernatant was lyophilized. In order to avoid proteoglycan degradation, the SNCE was not treated with acid, alkali, or by enzymolysis.

Quantity of glucuronic acid. Chondroitin sulfate (CS), a uronic acid, is a repeated sequence of D-glucuronic acid and $\mathrm{N}$-acetyl-D-galactosamine bound by sulfuric acid. The glucuronic acid levels of the fractions of SNCE or proteoglycan were quantified using the carbazole-sulfuric acid method, which has been used as a general method to determine CS or uronic acid levels (12).

Isolation of proteoglycan. Purified proteoglycan was pooled by repeating the following steps twelve times: first, $200 \mathrm{mg}$ of SNCE was dissolved in $20 \mathrm{ml}$ of $7 \mathrm{M}$ urea, $50 \mathrm{mM}$ Tris- $\mathrm{HCl}$, $\mathrm{pH} 7.4$ (7 M urea buffer) and applied to a DEAE-Sephacel ionexchange column $(\phi 5.0 \times 15 \mathrm{~cm}$; GE Healthcare Japan, Tokyo, Japan), equilibrated with $7 \mathrm{M}$ urea buffer and then eluted with a linear gradient of $0-1.0 \mathrm{M} \mathrm{NaCl}$ in the same solvent. DEAESephacel ion-exchange column chromatography was carried out at $4^{\circ} \mathrm{C}$, and $16-\mathrm{ml}$ fractions were collected. The glucuronic acid content in each fraction was determined by the carbazole sulfate method. The fractions positive for glucuronic acid and with an elution position corresponding to $\sim 0.5 \mathrm{M} \mathrm{NaCl}$ were regarded as CS proteoglycan and were pooled, desalted by ultrafiltration using Amicon stirred ultrafiltration cells (Millipore, Billerica, MA), and dried by lyophilization.

Molecular weight fractions of proteoglycan by gel filtration column chromatography. As molecular weight markers, three dextrans varying in molecular weight were prepared as follows: dextran from Leuconostoc mesenteroides (average MW 5,000,000-40,000,000; Sigma-Aldrich, St. Louis, MO) (D1); dextran standard 1,400,000 from L. mesenteroides (MW 1,400,000; Sigma-Aldrich) (D2); and dextran standard 270,000 from L. mesenteroides (MW 270,000; Sigma-Aldrich) (D3). Samples of $150 \mathrm{mg}$ of proteoglycan and $50 \mathrm{mg}$ of dextran markers (D1, D2, D3) were dissolved in $20 \mathrm{ml}$ of $0.1 \mathrm{M}$ phosphate buffer ( $\mathrm{pH} 7.1$ ) containing $0.2 \mathrm{M}$ sodium chloride $(0.1 \mathrm{M}$ phosphate buffer) and applied to a Sepharose CL-2B gel filtration column $(\phi 5.0 \times 50 \mathrm{~cm}$; GE Healthcare Japan) equilibrated with $0.1 \mathrm{M}$ phosphate buffer, and then eluted with the same buffer. Sepharose CL-2B gel filtration column chromatography was carried out at $4^{\circ} \mathrm{C}$, and $16-\mathrm{ml}$ fractions were collected.
Gel filtration column chromatography of proteoglycan was repeated six times. Next, the dextran contents of each dextran marker fraction were determined using the phenol-sulfuric acid method (13). D1 was used to measure the void volume $\left(\mathrm{V}_{0}\right)$. The optimal molecular weight separation range of dextran in Sepharose CL-2B was 100,000-20,000,000, and the $\mathrm{V}_{0}$ fraction was considered to have a molecular weight of 20,000,000. A standard curve of dextran molecular weight against fraction number was plotted based on measurements of dextran contents in D1, D2, and D3. The concentrations of glucuronic acid in each fraction were then determined using the carbazole-sulfuric acid method. The fraction positive for glucuronic acid was collected and divided into 3 portions according to the dextran standard curve as follows: $>5,000,000$ (PG-1), 400,000-5,000,000 (PG-2), <400,000 (PG-3). All three groups were desalted by ultrafiltration using Amicon stirred ultrafiltration cells and dried by lyophilization.

Animals. Male hairless mice (Hr-/Kud), 6 weeks old, were purchased from Kyudo Co., Ltd. (Fukuoka, Japan). Animals were fed a standard diet and water ad libitum. The mice were housed 6 in a cage and maintained at a constant temperature of $22 \pm 2^{\circ} \mathrm{C}$ with a $12 \mathrm{~h}$ light/dark cycle. Mice were 8 weeks old at the start of the experiment. All of the animal experiments in this study were carried out in accordance with the guidelines for animal experimentation of Hirosaki University.

Oral administration. Cages housing 6 mice were randomly allocated to receive one of the test sample solutions. The quantities of uronic acid in SNCE, PG-1, PG-2, and PG-3 were determined in advance, and samples containing $40 \mu \mathrm{g}$ of uronic acid in $0.5 \mathrm{ml}$ of distilled water were prepared. The mice were given $0.5 \mathrm{ml}$ of each sample or distilled water (nonirradiated control group and UVB-irradiated control group) 6 times per week using a stomach sonde needle $(\phi 1.2 \times 50 \mathrm{~mm})$. Administration of test samples was initiated three weeks prior to UVB irradiation and was continued for 11 weeks.

UVB irradiation for physiological and histological analysis. A bank of six 436-mm UVB lamps (GL15E; Sankyo Denki Co., Ltd., Kanagawa, Japan) was used. During the period of UVB irradiation, the mice were housed in a stainless steel irradiation chamber. The UVB lamp height was adjusted to deliver $1.0 \mathrm{~mW} / \mathrm{cm}^{2}$ at the dorsal surface of the mice. The time was adjusted to deliver the appropriate UVB daily irradiance. The mice were exposed to UVB irradiation 6 times per week, starting with $60 \mathrm{~mJ} / \mathrm{cm}^{2}$ as the minimal edematous dose (MED) for the first week, followed by $120 \mathrm{~mJ} / \mathrm{cm}^{2}$ (7 weeks) to constitute a total dose of $5.4 \mathrm{~J} / \mathrm{cm}^{2}$ over the 8 -week period. The UVB irradiance was measured using a radiometer (UVP UVX $310 \mathrm{~nm}$; UVP, LLC, Upland, CA).

Physiological analysis of the skin surface. An MPA 5 multiprobe analytical system (CK Electronic $\mathrm{GmbH}$, Cologne, Germany) was used for physiological analysis of the skin surface. The erythema value in the dorsal skin surface of hairless mice was measured using a Mexameter (MX 18; CK Electronic $\mathrm{GmbH})$. The TEWL $\left(\mathrm{g} / \mathrm{hm}^{2}\right)$ in the dorsal skin surface of hairless mice was measured using a Tewameter (TM300; CK Electronic GmbH) and the hydration level in 
the dorsal skin surface of hairless mice was measured using a Corneometer (CM825; CK Electronic $\mathrm{GmbH}$ ) as previously described. The measurements were performed after 4 and 7 weeks of UVB irradiation.

Histological analysis. Biopsy specimens were obtained from the central dorsal skin of hairless mice 1 day after the 8-week period of UVB irradiation. Skin biopsy samples were fixed in $18.5 \%$ formalin and embedded in paraffin. Serial sections of $3 \mu \mathrm{m}$ were cut and mounted onto silane-coated slide glasses (Matsunami Glass Ind. Ltd., Osaka, Japan). Skin sections were stained with hematoxylin and eosin (H\&E), and photomicrographs were captured under an inverted microscope (CKX41; Olympus Corporation, Tokyo, Japan) with a digital camera (CAMEDIA C-7070; Olympus Corporation). Epidermal and dermal thicknesses were measured at 10 sites in the photomicrographs of skin sections.

Inflammatory cytokine levels in serum and dorsal skin. Serum and dorsal skin biopsies were collected 1 day after the 8 weeks of UVB irradiation. The skin biopsy samples were frozen in liquid nitrogen, crushed using cell disruption equipment (Auto-Mill TK-AM5; Tokken, Inc., Chiba, Japan), and lyophilized. Freeze-dried samples $(15 \mathrm{mg})$ were suspended in $1 \mathrm{ml}$ of Dulbecco's phosphate buffered saline (SigmaAldrich) containing $0.05 \%$ (w/v) Triton X-100 and protease inhibitor cocktail (Complete Mini; Roche Diagnostics GmbH, Mannheim, Germany) for $30 \mathrm{~min}$ at $4^{\circ} \mathrm{C}$ and centrifuged at $12,000 \mathrm{rpm}$ for $30 \mathrm{~min}$ at $4^{\circ} \mathrm{C}$. After centrifugation, supernatants (skin extracts) were collected. Contents of inflammatory cytokines, tumor necrosis factor (TNF)- $\alpha$, interleukin (IL)-1 $\beta$, and IL-6, in serum and skin were determined using ELISA kits (Quantikine Immunoassay; R\&D Systems Inc., Minneapolis, $\mathrm{MN})$.

Statistical analysis. The results are shown as the means \pm SEM. Statistical analyses were performed using the Student's t-test. In all analyses, $\mathrm{p}<0.05$ was taken to indicate a statistically significant difference. Each experiment was repeated at least twice.

\section{Results}

Isolation of proteoglycan. Proteoglycan was isolated from SNCE by DEAE-Sephacel ion-exchange column chromatography equilibrated with $7 \mathrm{M}$ urea buffer and then eluted with a linear $\mathrm{NaCl}$ gradient from $0 \mathrm{M}$ to $1.0 \mathrm{M}$ in the same solvent. The elution profile of DEAE-Sephacel ion-exchange column chromatography is shown in Fig. 1. Twelve chromatography runs produced $\sim 1,086 \mathrm{mg}$ of proteoglycan from $2,400 \mathrm{mg}$ of SNCE (200 mg x 12).

Proteoglycan fractions of different molecular weights obtained by gel filtration column chromatography. Proteoglycan fractions of different molecular weights were isolated from the proteoglycan obtained by Sepharose CL-2B gel filtration column chromatography equilibrated with $0.1 \mathrm{M}$ phosphate buffer. The elution profile of Sepharose CL-2B gel filtration column chromatography is shown in Fig. 2. Elution peaks of glucuronic acid were confirmed near $\mathrm{V}_{0}$ and close

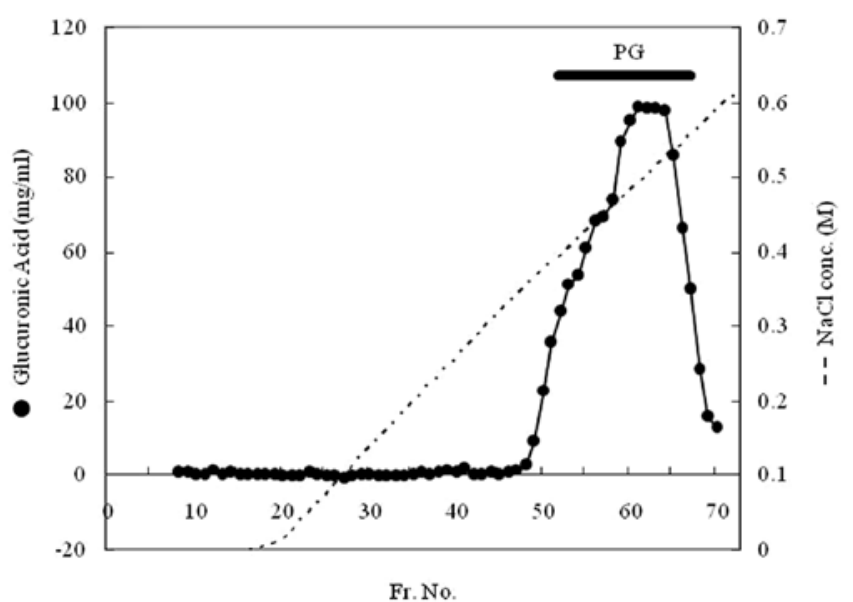

Figure 1. DEAE-Sephacel ion-exchange column chromatography of SNCE Samples of $200 \mathrm{mg}$ of SNCE were applied to a DEAE-Sephacel ion-exchange column $(\phi 5.0 \times 15 \mathrm{~cm}$; GE Healthcare Japan, Tokyo, Japan) equilibrated with $7 \mathrm{M}$ urea, $50 \mathrm{mM}$ Tris- $\mathrm{HCl}$ ( $\mathrm{pH} 7.4)$, and then eluted with a linear gradien of 0-1.0 M NaCl in the same solvent. DEAE-Sephacel ion-exchange column chromatography was carried out at $4^{\circ} \mathrm{C}$, and $16-\mathrm{ml}$ fractions were collected. The flow rate was $120 \mathrm{ml} / \mathrm{h}$. Fractions containing proteoglycan (PG) were pooled as indicated by the bar for further fractionation.

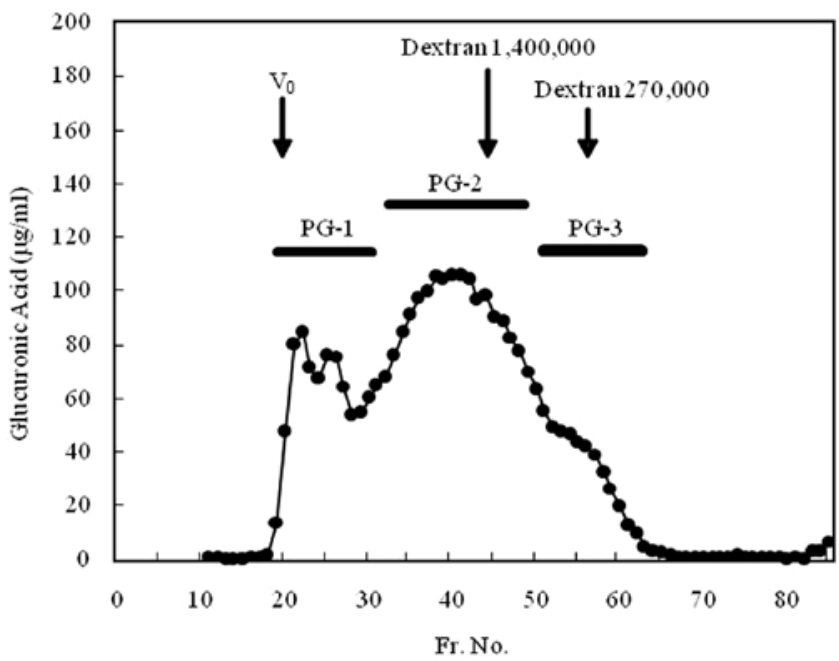

Figure 2. Sepharose CL-2B gel filtration column chromatography of the proteoglycan fraction. Samples of $150 \mathrm{mg}$ of proteoglycan were applied to a Sepharose CL-2B gel filtration column $(\phi 5.0 \times 50 \mathrm{~cm}$; GE Healthcare Japan $)$ equilibrated with $0.1 \mathrm{M}$ phosphate buffer ( $\mathrm{pH}$ 7.1) containing $0.2 \mathrm{M}$ sodium chloride, and then eluted with the same buffer. Sepharose CL-2B gel filtration column chromatography was carried out at $4^{\circ} \mathrm{C}$, and $16-\mathrm{ml}$ fractions were collected. The flow rate was $120 \mathrm{ml} / \mathrm{h}$. Arrows indicate the elution positions of void volume $\left(\mathrm{V}_{0}\right)$, dextran standard 1,400,000 (MW 1,400,000; SigmaAldrich, St. Louis, MO) (D2), and dextran standard 270,000 (MW 270,000; Sigma-Aldrich) (D3), respectively. PG-1, PG-2, and PG-3 were pooled as indicated by the bar. PG-1, proteoglycan (MW>5,000,000); PG-2, proteoglycan (MW>400,000-5,000,000); PG-3, proteoglycan (MW<400,000).

to the elution position of dextran standard 1,400,000. PG-1 $(>5,000,000)$, PG-2 (400,000-5,000,000), and PG-3 $(<400,000)$ were obtained as described in Materials and methods. Six chromatography runs produced $\sim 199 \mathrm{mg}$ of PG-1, 446 mg of PG-2, and $\sim 153 \mathrm{mg}$ of PG-3 from $900 \mathrm{mg}$ of proteoglycan (150 mg x 6). 

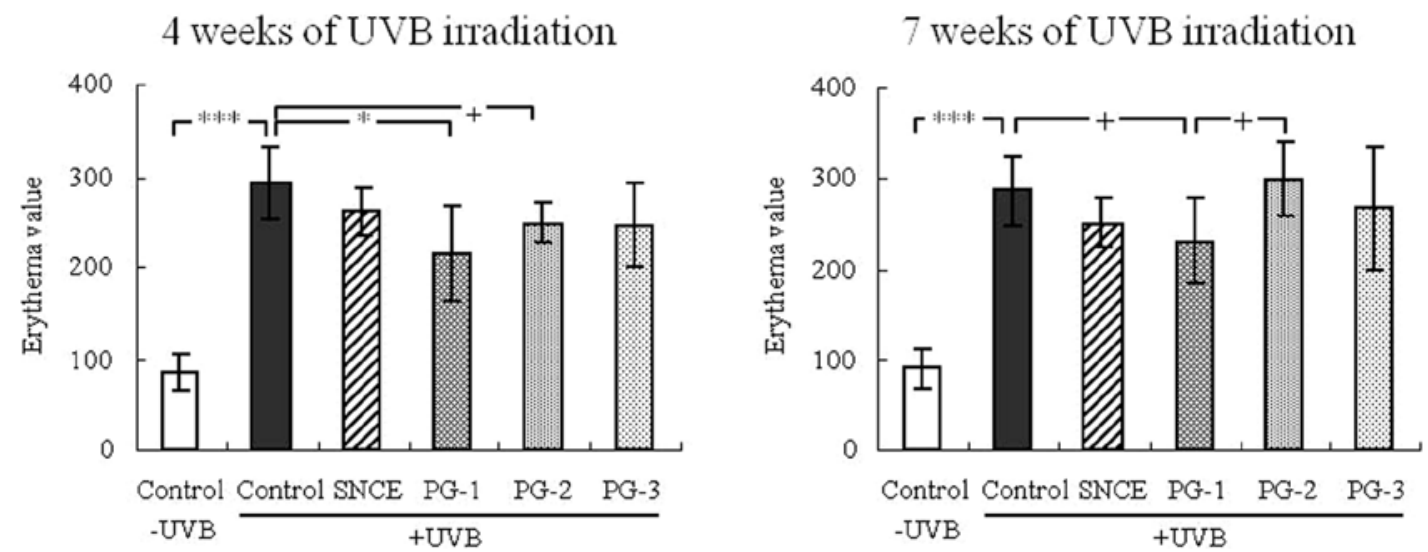

Figure 3. Erythema values in the dorsal skin surface of hairless mice was measured after 4 and 7 weeks of UVB irradiation. Values are the means \pm SEM. SNCE, salmon nasal cartilage extract; PG-1, proteoglycan (MW >5,000,000); PG-2, proteoglycan (MW>400,000-5,000,000); PG-3, proteoglycan (MW<400,000). *** $\mathrm{p}<0.001,{ }^{*} \mathrm{p}<0.05$, and ${ }^{+} \mathrm{p}<0.1$.

4 weeks of UVB irradiation

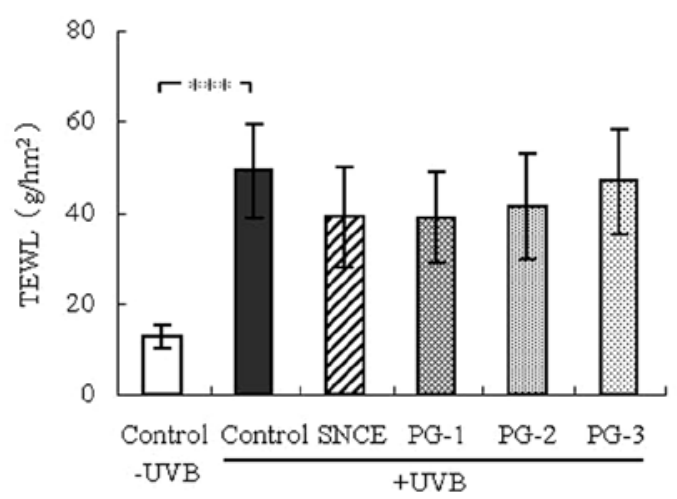

7 weeks of UVB irradiation

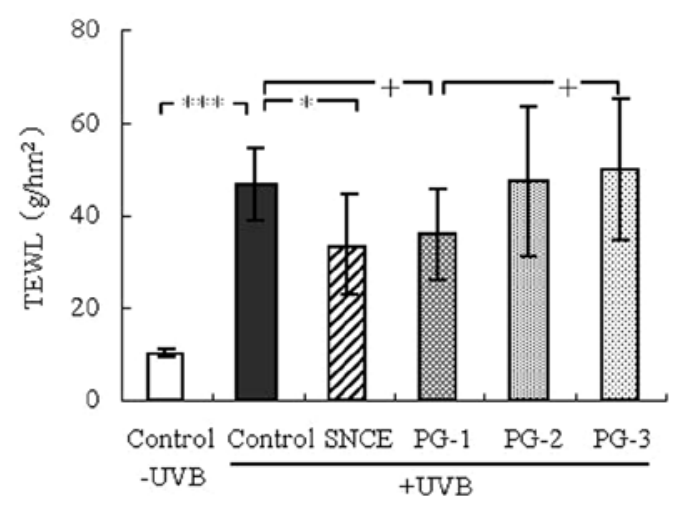

Figure 4. Transepidermal water loss (TEWL) in the dorsal skin surface of hairless mice was measured after 4 and 7 weeks of UVB irradiation. Values are the means \pm SEM. SNCE, salmon nasal cartilage extract; PG-1, proteoglycan (MW>5,000,000); PG-2, proteoglycan (MW>400,000-5,000,000); PG-3, proteoglycan $(\mathrm{MW}<400,000) .{ }^{* * *} \mathrm{p}<0.001,{ }^{*} \mathrm{p}<0.05$, and ${ }^{+} \mathrm{p}<0.1$.

Physiological analysis of the skin surface. The physiological aspects of the mouse dorsal skin surface following oral administration of SNCE and the various proteoglycan fractions were analyzed based on data obtained after 4 and 7 weeks of UVB irradiation. Erythema values, TEWL, and water contents of the stratum corneum are shown in Figs. 3-5, respectively. As shown in Fig. 3, UVB irradiation significantly increased the erythema value (non-irradiated and UVB-irradiated control groups). However, oral administration of PG-1 significantly inhibited the UVB-induced increase in the erythema value after 4 weeks of UVB irradiation $(\mathrm{p}<0.05)$. PG-1 also showed a slight but not statistically significant inhibitory effect on the UVB-induced increase in the erythema value after 7 weeks of UVB irradiation $(\mathrm{p}<0.1)$. Oral administration of $\mathrm{PG}-2$ showed a tendency to inhibit the UVB-induced increase in erythema value after 4 weeks of UVB irradiation $(p<0.1)$. In contrast, oral administration of PG-3 did not inhibit the increase in the erythema value associated with UVB irradiation. Results of the TEWL measurement are shown in Fig. 4. Similar to the erythema value, UVB irradiation significantly increased TEWL (non-irradiated control group and UVB-irradiated control group). However, SNCE significantly inhibited the
UVB-induced increase in TEWL, and PG-1 tended to restrain the UVB-induced increase in TEWL after 7 weeks of UVB irradiation $(\mathrm{p}<0.1)$. On the other hand, neither PG-2 nor PG-3 showed any effect on the UVB-induced increase in TEWL. As shown in Fig. 5, oral administration of SNCE significantly increased the hydration level, which was decreased by UVB irradiation, and PG-1 showed a tendency to increase the hydration level $(\mathrm{p}<0.1)$. However, the hydration level was unaffected by oral administration of PG-2 and PG-3.

Histological analysis. To examine the histological effects of oral administration of SNCE and proteoglycan fractions on mouse dorsal skin after 8 weeks of UVB irradiation, skin sections were stained with $\mathrm{H} \& \mathrm{E}$ and images were captured under a microscope (Fig. 6). The thickness of the epidermis and dermis was measured on photomicrographs, as shown in Table I. UVB irradiation for 8 weeks produced epidermal and dermal hypertrophies (non-irradiated control group and UVB-irradiated control group). Oral administration of SNCE and PG-1 markedly suppressed the UVB-associated increases in epidermal and dermal thicknesses compared with UVB-irradiated controls. Oral administration of PG-2 

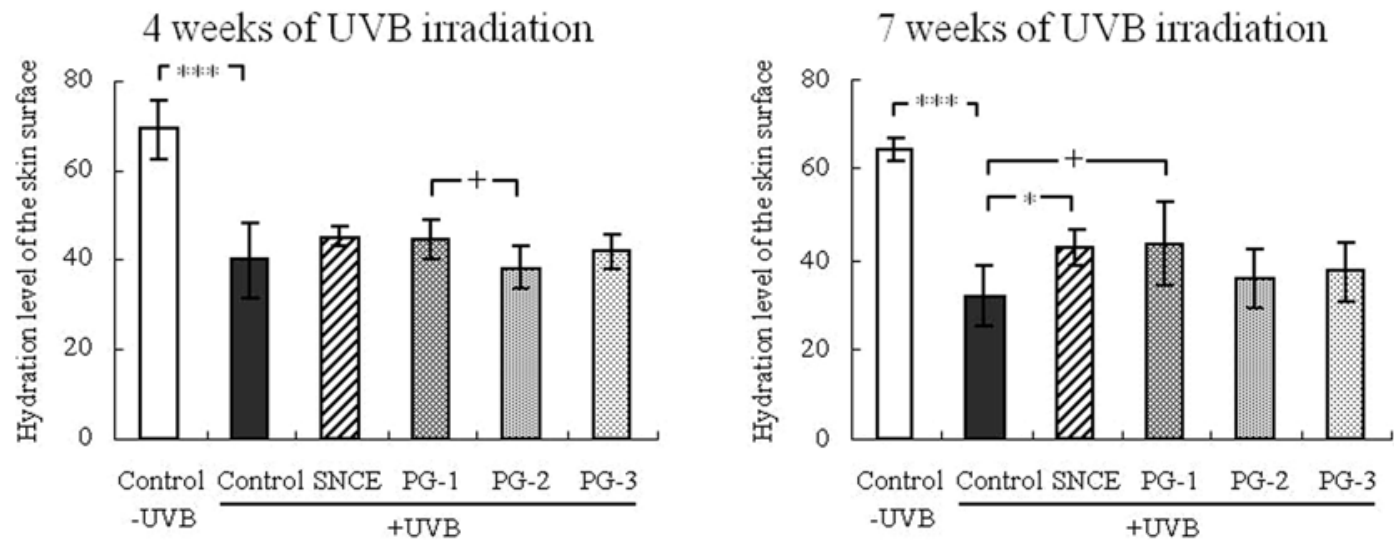

Figure 5. Hydration levels in the dorsal skin surface of hairless mice was measured after 4 and 7 weeks of UVB irradiation. Values are the means \pm SEM. SNCE, salmon nasal cartilage extract; PG-1, proteoglycan (MW >5,000,000); PG-2, proteoglycan (MW>400,000-5,000,000); PG-3, proteoglycan (MW<400,000). ${ }^{* * *} \mathrm{p}<0.001,{ }^{*} \mathrm{p}<0.05$, and ${ }^{+} \mathrm{p}<0.1$.
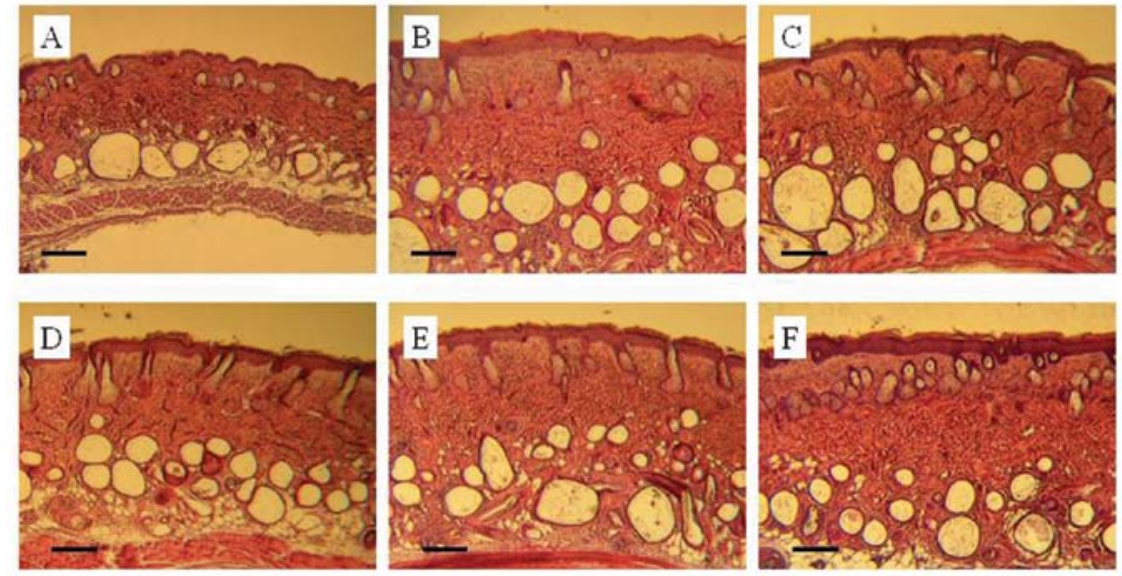

Figure 6. Biopsy specimens were obtained from the central dorsal skin of hairless mice 1 day after the 8-week period of UVB irradiation. Skin sections were stained with hematoxylin and eosin (H\&E) as described in Materials and methods. (A) Non-irradiated control. (B) UVB-irradiated control. UVB-irradiated specimens treated with (C) SNCE; (D) PG-1; (E) PG-2 and (F) PG-3. SNCE, salmon nasal cartilage extract; PG-1, proteoglycan (MW>5,000,000); PG-2, proteoglycan (MW>400,000-5,000,000); PG-3, proteoglycan (MW<400,000). Scale bar, $200 \mu$ m. Magnification, $x 40$.

tended to suppress the UVB-associated increase in epidermal thickness compared with UVB-irradiated controls, but had no effect on the thickness of the dermis. On the other hand, oral administration of PG-3 showed no effect on UVB-induced hypertrophy of either the epidermis or dermis.

Inflammatory cytokine levels in serum and dorsal skin. UVB irradiation was shown to increase the contents of the inflammatory cytokines, TNF- $\alpha$, IL- $1 \beta$, and IL-6, in serum (Fig. 7A) and skin (Fig. 7B) (non-irradiated control group and UVB-irradiated control group). Although differences were not always significant, oral administration of high molecular weight proteoglycan fractions from salmon nasal cartilage downregulated the excess serum and skin levels of all three cytokines associated with UVB irradiation in a molecular weight-dependent manner.

\section{Discussion}

In our previous study (11), we demonstrated that oral intake of SNCE containing high levels of proteoglycan can prevent aging of skin in a hairless mouse model of UVB-induced skin aging. However, as a crude extract was used in these experiments, the active ingredient of SNCE could not be identified. In the present study, we first extracted proteoglycan from SNCE by DEAE-Sephacel ion-exchange column chromatography. The proteoglycan thus obtained was fractionated into different molecular weights by Sepharose CL-2B gel filtration column chromatography, and the effects of three different proteoglycan fractions were evaluated in a model of skin aging involving exposure of hairless mice to repeated UVB irradiation.

The erythema values, TEWL, and water contents of the stratum corneum were determined as physiological measures of the skin surface, and epidermal and dermal hypertrophies were measured by histological analysis. The results indicated that the effects of oral administration of high molecular weight proteoglycan were molecular weight-dependent in all evaluations. Erythema reflects the inflammatory response induced by UVB exposure $(14,15)$. The TEWL value can be regarded as an indicator of epidermal barrier function $(16,17)$, and water contents of the stratum corneum reflect cutaneous dryness. Moreover, epidermal and dermal hypertrophies are 
Table I. Epidermal and dermal thickness measured on photomicrographs of the skin sections. ${ }^{\mathrm{a}}$

\begin{tabular}{|c|c|c|c|c|c|}
\hline \multirow[b]{2}{*}{ Sample } & \multirow[b]{2}{*}{$\begin{array}{l}\text { UVB } \\
\text { irradiation }\end{array}$} & \multicolumn{2}{|c|}{ Epidermis } & \multicolumn{2}{|c|}{ Dermis } \\
\hline & & $\begin{array}{l}\text { Thickness } \\
(\mu \mathrm{m})\end{array}$ & $\begin{array}{c}\text { Significance } \\
\text { (vs. control + UVB) }\end{array}$ & $\begin{array}{l}\text { Thickness } \\
\qquad(\mu \mathrm{m})\end{array}$ & $\begin{array}{c}\text { Significance } \\
\text { (vs. control + UVB) }\end{array}$ \\
\hline Control & - & $18.8 \pm 3.0$ & $* * *$ & $292 \pm 28$ & $* * *$ \\
\hline Control & + & $72.1 \pm 2.0$ & & $467 \pm 20$ & \\
\hline SNCE & + & $56.8 \pm 6.0$ & $*$ & $401 \pm 45$ & $*$ \\
\hline PG-1 & + & $62.4 \pm 5.8$ & $* *$ & $396 \pm 15$ & $* * *$ \\
\hline PG-2 & + & $65.1 \pm 6.3$ & + & $422 \pm 88$ & NS \\
\hline PG-3 & + & $74.3 \pm 7.9$ & NS & $458 \pm 34$ & NS \\
\hline
\end{tabular}

${ }^{a}$ Measurements were obtained from photomicrographs shown in Fig. 6. Values are the means \pm SEM. SNCE, salmon nasal cartilage extract; PG-1, proteoglycan (MW $>5,000,000)$; PG-2, proteoglycan (MW $>400,000-5,000,000)$; PG-3, proteoglycan $(\mathrm{MW}<400,000)$; NS, not significant. ${ }^{* * *} \mathrm{p}<0.001,{ }^{* *} \mathrm{p}<0.01,{ }^{*} \mathrm{p}<0.05$, and ${ }^{+} \mathrm{p}<0.1$.

A
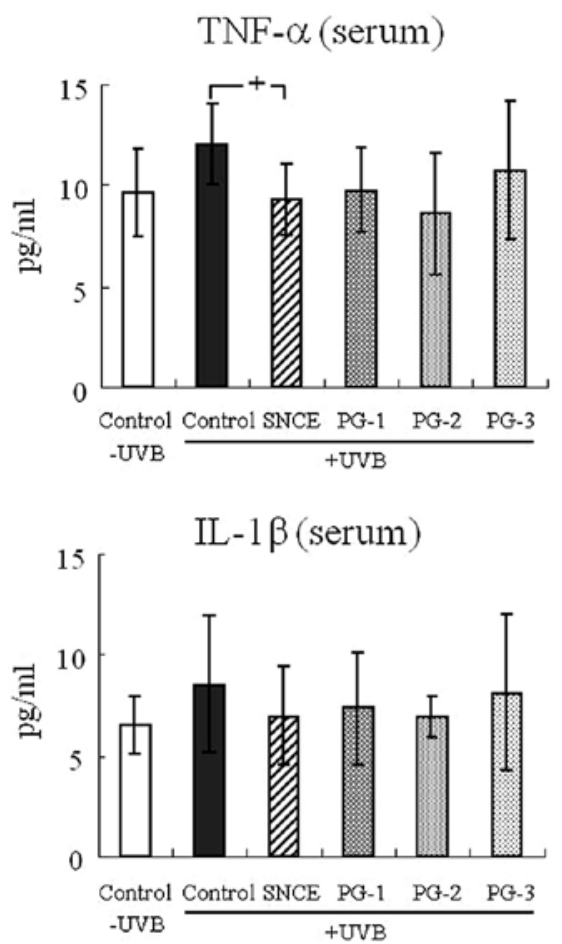

IL-6 (serum)

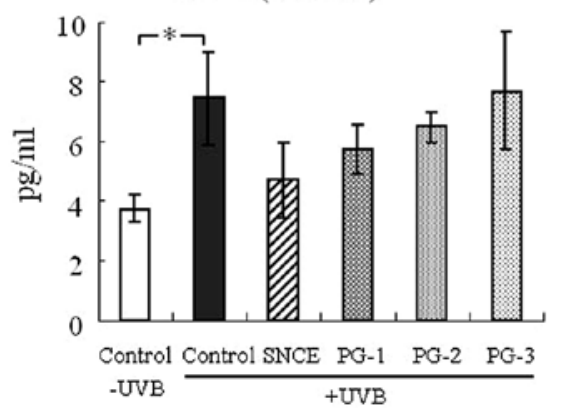

B skin
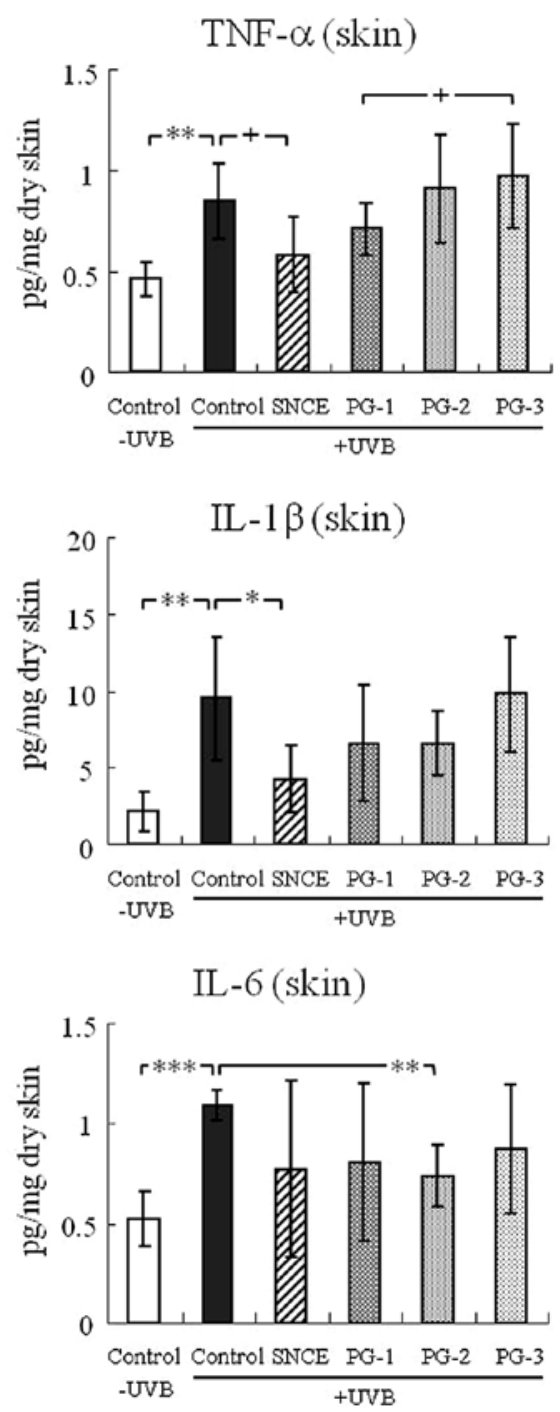

Figure 7. Inflammatory cytokine levels in (A) serum and (B) dorsal skin. Serum and dorsal skin biopsies were collected 1 day after the 8-week period of UVB irradiation as described in Materials and methods. Values are the means \pm SEM. SNCE, salmon nasal cartilage extract; PG-1, proteoglycan (MW $>5,000,000)$; PG-2, proteoglycan (MW $>400,000-5,000,000)$; PG-3, proteoglycan $(\mathrm{MW}<400,000) .{ }^{* * * *} \mathrm{p}<0.001,{ }^{* *} \mathrm{p}<0.01,{ }^{*} \mathrm{p}<0.05$, and ${ }^{+} \mathrm{p}<0.1$. 
associated with loss of cutaneous flexibility. Therefore, oral intake of high molecular weight proteoglycan is expected to show anti-aging effects on the skin, including suppression of UVB-induced inflammation, normalization of the epidermal barrier, prevention of cutaneous dryness, and preservation of skin flexibility.

Aggrecan, the largest proteoglycan with a molecular weight of $2,200 \mathrm{kDa}$, is abundant in cartilage and the complete coding sequences for aggrecan core protein have been determined in the rat, mouse, human, and chicken (18). Vincent et al reported that proteoglycan of bovine nasal cartilage contains $87 \% \mathrm{CS}$, $6 \%$ keratan sulfate, and $7 \%$ protein (19). There have been no previous studies regarding the structure of proteoglycan in salmon nasal cartilage. However, Kakizaki et al analyzed the core protein of salmon nasal cartilage proteoglycan by nano-LC/MS/MS, and reported that the most common salmon nasal cartilage proteoglycan was aggrecan and that the GAG chains bound to the core protein of salmon nasal cartilage proteoglycan were comprised of CS (20). In addition, aggrecan was shown to bind to hyaluronic acid in vivo and to form very large aggregates (21-25).

In the present study, the detailed structures of PG-1, PG-2, and PG-3 were not confirmed. However, on the basis of the molecular weight, it was assumed that PG-2 (400,0005,000,000: in terms of dextran) was aggrecan. In DEAE deionization column chromatography using $7 \mathrm{M}$ urea buffer, proteoglycan was likely not associated with hyaluronic acid. However, contrary to prior expectations, the structure of PG-1 may be an aggregate consisting of proteoglycan and hyaluronic acid or an aggregate of proteoglycan and collagen. It was assumed that PG-3 ( $<400,000$ : in terms of dextran) was a degradation product of aggrecan resolved by enzymes, such as proteases, and not raw aggrecan. More detailed analyses are necessary in future studies.

The anti-aging effects on the skin were molecular weightdependent. In our previous study (11), CS, which is a degradation product of salmon nasal cartilage proteoglycan, did not show any beneficial effects in UVB-induced photoaging. Therefore, the skin anti-aging effects of oral proteoglycan may disappear with decomposition of the proteoglycan. In addition, it is not possible for high molecular weight proteoglycan to be absorbed via the gastrointestinal tract.

Sashinami et al reported that proteoglycan from salmon nasal cartilage modulates cytokine responses to Escherichia coli in mouse macrophage (26). UVB irradiation induces inflammatory cytokines, such as interleukin-1, interleukin-6, and TNF- $\alpha$ (27-32). Inflammation induced by UVB irradiation produces erythema (14) and subsequently leads to cutaneous hypertrophy (31). In the present study, oral administration of high molecular weight proteoglycan showed an inhibitory effect on excessive inflammatory cytokine production by UVB irradiation in a molecular weight-dependent manner.

In conclusion, we demonstrated that high molecular weight proteoglycan from salmon nasal cartilage, consisting of aggrecan or aggregates of aggrecan, acts on gut immunity and improves skin condition by inhibiting surplus inflammatory cytokine production induced by UVB irradiation. The high molecular weight proteoglycan from salmon nasal cartilage may serve as an anti-aging agent to achieve healthy skin.

\section{Acknowledgements}

This study was supported in part by the City Area Program for the Promotion of Science and Technology in Regional Areas from the Ministry of Education, Culture, Sports, Science, and Technology of Japan, and by a grant for the Promotion of International Scientific Research (2008, C-1), Japan.

\section{References}

1. Holleran WM, Uchida Y, Halkier-Sorensen L, Haratake A Hara M, Epstein JH and Elias PM: Structural and biochemical basis for the UVB-induced alterations in epidermal barrier function. Photodermatol Photoimmunol Photomed 13: 117-128, 1997.

2. Abe T and Mayuzumi J: The change and recovery of human skin barrier functions after ultraviolet light irradiation. Chem Pharm Bull (Tokyo) 27: 458-462, 1979.

3. Bissett DL, Hannon DP and Orr TV: An animal model of solar-aged skin: histological, physical, and visible changes in UV-irradiated hairless mouse skin. Photochem Photobiol 46: 367-378, 1987.

4. Matsuda N, Koyama Y, Hosaka Y, Ueda H, Watanabe T, Araya T, Irie $S$ and Takehana K: Effects of ingestion of collagen peptide on collagen fibrils and glycosaminoglycans in the dermis. J Nutr Sci Vitaminol (Tokyo) 52: 211-215, 2006.

5. Tanaka M, Koyama Y and Nomura Y: Effects of collagen peptide ingestion on UV-B-induced skin damage. Biosci Biotechnol Biochem 73: 930-932, 2009.

6. Kim SY, Kim SJ, Lee JY, Kim WG, Park WS, Sim YC and Lee SJ: Protective effects of dietary soy isoflavones against UV-induced skin-aging in hairless mouse model. J Am Coll Nutr 23: 157-162, 2004.

7. Vayalil PK, Mittal A, Hara Y, Elmets CA and Katiyar SK: Green tea polyphenols prevent ultraviolet light-induced oxidative damage and matrix metalloproteinases expression in mouse skin. J Invest Dermatol 122: 1480-1487, 2004.

8. Poole AA: Proteoglycans in health and disease: structures and functions. J Biol Chem 236: 1-14, 1986.

9. Mitsui T, Sashinami H, Sato F, Kijima H, Ishiguro Y, Fukuda S, Yoshihara S, Hakamada K and Nakane A: Salmon cartilage proteoglycan suppresses mouse experimental colitis through induction of Foxp $3^{+}$regulatory $\mathrm{T}$ cells. Biochem Biophys Res Commun 402: 209-215, 2010.

10. Ota S, Yoshihara S, Ishido K, Tanaka M, Takagaki K and Sasaki M: Effects of proteoglycan on dextran sulfate sodiuminduced experimental colitis in rats. Dig Dis Sci 53: 3176-3183, 2008.

11. Goto $M$, Ito S, Kato Y, Yamazaki S, Yamamoto $\mathrm{K}$ and Katagata Y: Anti-aging effects of extracts prepared from salmon nasal cartilage in hairless mice. Mol Med Rep 4: 779-784, 2011.

12. Bitter T and Muir HM: A modified uronic acid carbazole reaction. Anal Biochem 4: 330-334, 1962.

13. Hodge JE and Hofreiter BT: Determination of reducing sugars and carbohydrates. In: Methods in Carbohydrate Chemistry. Vol. 1. Whistler RI, Wolfrom MI (eds.) Academic Press, New York, pp380-391, 1962.

14. Nose T and Tsurumi K: Pharmacological studies on cutaneous inflammation induced by ultraviolet irradiation (1): Quantification of erythema by reflectance colorimetry and correlation with cutaneous blood flow. Jpn J Pharmacol 62: 245-256, 1993.

15. Clydesdale GJ, Dandie GW and Muller HK: Ultraviolet light induced injury: immunological and inflammatory effects. Immunol Cell Biol 79: 547-568, 2001.

16. Abe T: Studies on skin surface barrier functions. Transepidermal water loss and skin surface lipids during childhood. Chem Pharm Bull (Tokyo) 26: 1659-1665, 1978.

17. Baker $\mathrm{H}$ : The effects of dimethylsulfoxide dimethylformamide and dimethylacetamide on the cutaneous barrier to water in human skin. J Invest Dermatol 50: 283-288, 1968.

18. Watanabe H, Yamada Y and Kimata K: Roles of aggrecan, a large chondroitin sulfate proteoglycan, in cartilage structure and function. J Biochem 124: 687-693, 1998.

19. Hascall VC and Sajdera SW: Physical properties and polydispersity of proteoglycan from bovine nasal cartilage. J Biol Chem 245: 4920-4930, 1970. 
20. Kakizaki I, Tatara Y, Majima M, Kato Y and Endo $\mathrm{M}$ : Identification of proteoglycan from salmon nasal cartilage. Arch Biochem Biophys 506: 58-65, 2011.

21. Hardingham TE and Muir H: Hyaluronic acid in cartilage and proteoglycan aggregation. Biochem J 139: 565-581, 1974

22. Buckwalter JA and Rosenberg LC: Electron microscopic studies of cartilage proteoglycans. Direct evidence for the variable length of the chondroitin sulfate-rich region of proteoglycan subunit core protein. J Biol Chem 257: 9830-9839, 1982.

23. Mörgelin M, Paulsson M, Hardingham TE, Heinegård D and Engel J: Cartilage proteoglycans. Assembly with hyaluronate and link protein as studied by electron microscopy. Biochem J 253 : $175-185,1988$

24. Iozzo RV and Murdoch AD: Proteoglycans of the extracellular environment: clues from the gene and protein side offer novel perspectives in molecular diversity and function. FASEB $\mathrm{J} 10$ : 598-614, 1996.

25. Kiani C, Chen L, Wu YJ, Yee AJ and Yang BB: Structure and function of aggrecan. Cell Res 12: 19-32, 2002.

26. Sashinami H, Takagaki K and Nakane A: Salmon cartilage proteoglycan modulates cytokine responses to Escherichia coli in mouse macrophages. Biochem Biophys Res Commun 351: 1005-1010, 2006.
27. Oxholm A, Oxholm P, Staberg B and Bendtzen K: Immunohistological detection of interleukin I-like molecules and tumour necrosis factor in human epidermis before and after UVB-irradiation in vivo. Br J Dermatol 118: 369-376, 1988.

28. Urbanski A, Schwarz T, Neuner P, Krutmann J, Kirnbauer R, Köck A and Luger TA: Ultraviolet light induces increased circulating interleukin-6 in humans. J Invest Dermatol 94: 808-811, 1990.

29. Nishimura N, Tohyama C, Satoh M, Nishimura H and Reeve VE: Defective immune response and severe skin damage following UVB irradiation in interleukin-6-deficient mice. Immunology 97: 77-83, 1999.

30. Nishimura N, Reeve VE, Nishimura H, Satoh M and Tohyama C: Cutaneous metallothionein induction by ultraviolet $\mathrm{B}$ irradiation in interleukin-6 null mice. J Invest Dermatol 114: 343-348, 2000.

31. Kobayashi S: UVB-induced skin damage and the protection/ treatment-effects of a novel, hydrophilic gamma-tocopherol derivative. Yakugaku Zasshi 126: 677-693, 2006 (In Japanese).

32. Osborne-Hereford AV, Rogers SW and Gahring LC: Neuronal nicotinic alpha7 receptors modulate inflammatory cytokine production in the skin following ultraviolet radiation. J Neuroimmunol 193: 130-139, 2008. 